\title{
Benefits and Drawbacks of Reference Architectures
}

\author{
Silverio Martínez-Fernández ${ }^{1}$, Claudia Ayala ${ }^{1}$, Xavier Franch $^{1}$, Helena Martins \\ Marques $^{2}$ \\ ${ }^{1}$ GESSI Research Group, Universitat Politècnica de Catalunya, Barcelona, Spain \\ \{smartinez, cayala, franch\}@essi.upc.edu \\ 2 everis, Barcelona, Spain \\ hmartinmeeveris.com
}

\begin{abstract}
Reference architectures (RA) have been studied to create a consistent notion of what constitutes them as well as their benefits and drawbacks. However, few empirical studies have been conducted to provide evidence that support the claims made. To increase this evidence, this paper investigates the actual industrial practice of using RAs. The study consists of a survey with 28 stakeholders from everis, a multinational consulting company based in Spain. We report the findings and contextualize them with previous research.
\end{abstract}

Keywords. Software reference architecture, empirical software engineering

\section{Introduction}

Software reference architectures (RA) have emerged as an approach to guide the development, standardization and evolution of concrete software architectures for new systems [5]. As in [1], we refer to the definition of RA as stated by Bass et al.: "a reference model mapped onto software elements and the data flows between them".

RAs have become widely studied and used in research and practice [1], as they are claimed to increase speed, reduce operational expenses and improve quality in software systems development mainly due to reuse [6]. Nonetheless, limited evidence exists to support these claims [7]. Therefore, the goal of this study is to investigate:

How practitioners perceive the potential benefits and drawbacks of RAs?

Industrial context. This study is part of an ongoing action-research initiative among everis and our research group, aimed to improve everis' architectural practices. everis is a software consulting company that offers solutions for big businesses that provide a wide spectrum of services to their customers. The solution that everis provides them is based on the deployment of an RA in their company, from which concrete software architectures are derived and used in a wide spectrum of applications. In this context, everis commissioned our research group to systematically gather empirical evidence about the benefits and drawbacks of the adoption of RAs for their clients, in order to avoid just relying on anecdotal evidences.

Martínez, S. [et al.]. Benefits and drawbacks of reference architectures. A: European Congress on Software Architecture. "Software Architecture, 7th European Conference, ECSA 2013: Montpellier, France, July 1-5, 2013: proceedings". Springer, 2013, p. 307-310.

The final authenticated version is available online at https://doi.org/10.1007/978-3-642-39031-9_26 


\section{Benefits and drawbacks of RAs from the Literature}

We identified the following benefits (B) and drawbacks (D) from the literature:

- (B1) Standardization of concrete architectures of systems [1][4][5][7][8].

- (B2) Facilitation of the design of concrete architectures for system development and evolution [1][5], improving the productivity of system developers [3][4][8].

- (B3) Systematic reuse of common functionalities and configurations in systems generation [2][4][5], implying shorter time-to-market and reduced cost [3][4].

- (B4) Risk reduction through the use of proven and partly prequalified architectural elements [2][4].

- $\quad$ (B5) Better quality by facilitating the achievement of quality attributes [3][8].

- (B6) Interoperability of different systems [2][4][5].

- (B7) Creation of a knowledge repository that allows knowledge transfer [2][7].

- (B8) Flexibility and a lower risk in the choice of multiple suppliers [2].

- (B9) Elaboration of the organization mission, vision and strategy [2].

- (D1) The need for an initial investment [6].

- (D2) Inefficient instantiation for the organization's systems [5].

\section{Benefits and Drawbacks of RAs from our Study}

9 RA projects executed in 9 different organizations that were clients of everis were analyzed. 28 stakeholders from these projects participated in the study. They covered 3 essential roles: 9 software architects and 9 architecture developers that designed and implemented RAs for the 9 client organizations; and 10 application builders who created RA-based applications. We report the benefits and drawbacks of RAs for the development of systems as seen by these practitioners. Fig. 1 includes a bar chart that shows the frequency in which stakeholders mentioned RA benefits and drawbacks. The reader is encouraged to see how this study was conducted in www.essi.upc.edu/ gessi/papers/ecsa13-annex.pdf .

Main benefits of RA adoption. We report benefits for RA acquisition organizations with the code "Ben" whereas we use the code "Ven" for the benefits to RA vendors.

- (Ben-A) Reduced development costs. Mainly due to software component reuse that facilitate functionality and speed up the process, leading to shorter time-to-market.

- (Ben-B) Reduced maintenance costs. Because of: better understandability of systems derived from the RA; the fact that RA common elements have fewer errors.

- (Ben-C) Easier development and increased productivity of application builders by architecturally-significant requirements already addressed and RA artifacts.

- (Ben-D) Incorporation of latest technologies, which among other things facilitates the recruitment of professionals with the required technological skills.

- (Ben-E) Applications more aligned with business needs.

- (Ben-F) Homogenization (or standardization) of the development and maintenance of a family of applications by defining procedures and methodologies. 
- (Ben-G) Increased reliability of RAs software elements, which are common for applications, that have been tested and matured, with the reliability that it implies.

- (Ben-H) Others benefits.

- (Ven-A) The consulting company harvests experience for prospective RA projects. The main reason is that requirements are very similar between client organizations.

- (Ven-B) Reusing architectural knowledge can speed up prospective RA projects and reduce time-to-market (e.g., by reducing their planning and development time).

- (Ven-C) They gain reputation for prospective client organizations and gain organizational competence.

- (Ven-D) Previous experience reduces the risks in future projects because a "to-be" model exists. It can be used in projects without very specific requirements.

- (Ven-E) It provides a shared architectural mindset.

- (Ven-F) It turns tacit knowledge into explicit knowledge in the reference model. Some tool support (e.g., wiki technologies) helps in managing such knowledge.

Main drawbacks of using RAs.

- (Dra-A) Additional high or medium learning curve for using the RA features.

- (Dra-B) Limited innovation by giving prescriptive guidelines for applications.

- (Dra-C) Applications' dependency over the RA. When applications have requirements that the RA does not offer yet, applications development is stopped.

- (Dra-D) Complexity. Participants who indicated that the use of the RA is complex.

- (Dra-E) None. Responders who indicated that RA adoption presents no drawbacks.

- (Dra-F) Wrong decisions about the technologies to be used in all the applications.

- (Dra-G) Other drawbacks.

\section{Discussion of Main Findings and Conclusions}

Table 1 summarizes the benefits and drawbacks of RAs respectively. Its columns respectively indicate: 1 ) benefits or drawbacks from the literature and uncovered ones by our survey that we could not match to the former ones; 2) the extent to which the results from our survey confirm $(\sqrt{ })$, partially support or help to understand $( \pm)$, do not explicitly mention $\left({ }^{\circ}\right)$, refuse theoretical evidences $(x)$ or uncover new results (new); and 3) survey findings related to such benefits or drawbacks.

In conclusion, a survey was conducted to analyze benefits and drawbacks of RAs in the industrial practice. It provides evidence to corroborate or refuse existing research. The main findings were: 1) the support of already known RAs benefits, mainly cost savings in the development and evolution of software systems, and the facilitation of the design of concrete software architectures; 2) new risks of adopting RAs emerged, such as additional learning curve, less room for innovation and complexity. As future work, we plan to perform further analysis of this survey.

Acknowledgements. This work has been supported by "Cátedra everis" and the Spanish project TIN2010-19130-C02-00. We thank all participants of the survey. 


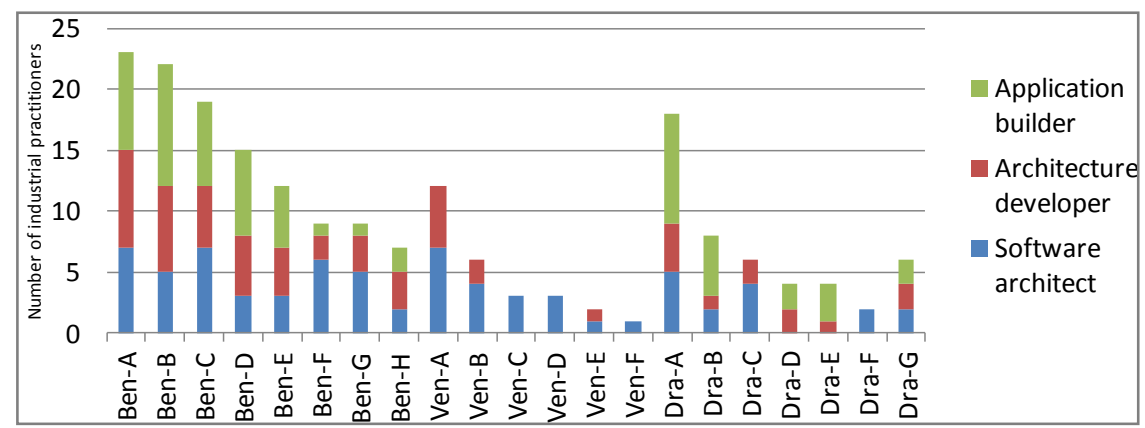

Fig. 1. Benefits and Drawbacks of RA adoption in organizations as seen by practitioners.

Table 1. Summary of benefits and drawbacks of RAs.

\begin{tabular}{|r|c|l||r|l|l|}
\hline \multicolumn{1}{|c|}{ Benefits } & $\mathbf{D}^{\mathbf{1}}$ & \multicolumn{1}{|c||}{ Findings } & Drawbacks & $\mathbf{D}^{\mathbf{1}}$ & Findings \\
\hline Standardization (B1) & $\sqrt{ }$ & Ben-F & Investment (D1) & \pm & Dra-G \\
\hline Facilitation (B2) & $\sqrt{ }$ & Ben-C & $\begin{array}{r}\text { Inefficient } \\
\text { instantiation (D2) }\end{array}$ & \pm & Imp-C \\
\hline Reuse (B3) & $\sqrt{ }$ & $\begin{array}{l}\text { Ben-A, Ben-B, } \\
\text { Ven-B }\end{array}$ & Learning curve & new & Dra-A \\
\hline Risk reduction (B4) & $\sqrt{ }$ & Ben-G, Ven-D & Limited innovation & new & Dra-B \\
\hline Enhanced quality (B5) & \pm & Ben-E & RA dependency & new & Dra-C \\
\hline Interoperability (B6) & $\circ$ & & Complexity & new & Dra-D \\
\hline Knowledge repository (B7) & $\sqrt{ }$ & Ven-A, Ven-F & Wrong decisions & new & Dra-F \\
\hline Flexibility for suppliers (B8) & \pm & Ven-E & & & \\
\hline Mission, vision, strategy (B9) & $\times$ & 3 & & & \\
\hline Latest technologies used & new & Ben-D & & \\
\hline Reputation & new & Ven-C & & \\
\hline
\end{tabular}

a. Notes: 1) diagnostic; 2) not mentioned as a benefit; 3) mentioned as enterprise architecture benefit.

\section{References}

1. Angelov, S., Grefen, P., Greefhorst, D.: A framework for analysis and design of software reference architectures. Information and Software Technology 54(4), 417 - 431 (2012).

2. Cloutier, R., Muller, G., Verma, D., Nilchiani, R., Hole, E., Bone, M.: The concept of reference architectures. Systems Engineering 13(1), 14-27 (2010)

3. Dobrica, L., Ovaska, E.: Analysis of a Cross-Domain Reference Architecture using Change Scenarios. SAVA@ECSA, (2011)

4. Gallagher, B.: Using the architecture tradeoff analysis method sm to evaluate a reference architecture: A case study. SEI CMU Tech. rep., DTIC Document (2000)

5. Galster, M., Avgeriou, P.: Empirically-grounded reference architectures: a proposal. QoSA-ISARCS, pp. 153-158 (2011)

6. Martínez-Fernández, S, Ayala, C., Franch, X., Martins, H.: REARM: A Reused-Based Economic Model for Software Reference Architectures. ICSR (2013)

7. Muller, G., Laar, P.: Researching reference architectures. CSER (2009)

8. Nakagawa, E., Oliveira, P., Becker, M.: Reference architecture and product line architecture: A subtle but critical difference. ECSA, pp. 207-211 (2011) 